\title{
MULTIPLIERS ON THE RIGID MOTIONS OF THE PLANE AND THEIR RELATIONS TO MULTIPLIERS ON DIRECT PRODUCTS
}

\author{
RICHARD L. RUBIN
}

\begin{abstract}
Hörmander multiplier theorems on $M(2)$ and $R^{2} \times T$ are developed. The relations between these theorems are studied. Applications to fractional Laplace operators and $R^{3}$ Riesz transforms are given.
\end{abstract}

The purpose of this paper is to study Hörmander multipliers on the nonabelian group of rigid motions of the Euclidean plane and to show how these multipliers correspond to Hörmander multipliers on the direct product of the plane with the circle. The relationships established may be used to demonstrate how aspects of harmonic analysis on the motion group arise from harmonic analysis on three-dimensional Euclidean space. The primary tool used in the study of Hörmander multipliers in this paper is the theory of spaces of homogeneous type developed by Coifman, de Guzman, and Weiss (cf. [1]).

The author wishes to thank Professors Guido Weiss and Ronald Coifman for their many helpful discussions concerning this work.

1. We review the notation essential to analysis on the motion group. For details of the elements of Fourier analysis on this group see [4]. The threedimensional nonabelian solvable group of rigid motions of the Euclidean plane, denoted by $M(2)$, consists of transformations, $(x, \phi)$, which map $y \in C$ to $(x, \phi)(y) \in C$ where $(x, \phi)(y)=e^{i \phi} y+x$, for $x \in C, \phi \in R^{2} / 2 \pi Z=T$. The Fourier transform on $M(2)$ is the linear transformation which maps $f \in L^{p}(M(2)) \cap L^{2}(M(2))$ to the operator-valued function $T_{f}(R)$ on $(0, \infty)$ defined as follows: if $g \in L^{2}(T)$, then for each $R>0$,

$$
\left[T_{f}(R)\right] g(\theta)=\int_{-\pi}^{\pi} f\left(\widehat{R e^{i(\theta+\phi)}}, \phi\right) g(\theta+\phi) d \phi
$$

where

$$
f(\hat{z}, \phi)=\frac{1}{2 \pi} \int_{R^{2}} f(x, \phi) e^{-i x \cdot z} d x .
$$

By considering the matrix of $T_{f}(R)$ with respect to the basis $\left\{e^{i k \theta}: k\right.$ is an integer $\}$, to be the matrix with entries $T_{f}(R, j, k)$ we obtain

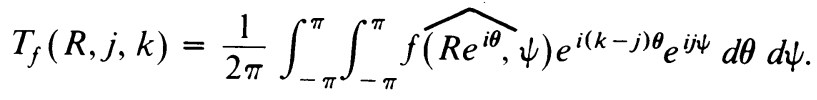

Received by the editors July 7, 1975.

AMS (MOS) subject classifications (1970). Primary 43A80, 42A18.

(c) American Mathematical Society 1976 
Given any function $f \in L^{2}(M(2))$, we may recover $f$ from its matricial Fourier transform by the formula

$$
f\left(r e^{i \phi}, \psi\right)=\frac{1}{2 \pi} \int_{0}^{\infty} \operatorname{tr}\left[T_{f}(R) J(\phi, r R, \psi)\right] R d R
$$

where $J(\phi, r, \psi)$ is the matrix with $j, k$ entry $i^{j-k} J_{j-k}(r) e^{-i[(j-k) \phi+k \psi]}$ and equality is in $L^{2}(M(2))$. Here $J_{n}(x)$ denotes the Bessel function of the first kind of order $n$ and tr denotes the trace operator. We also have the Plancherel formula:

$$
\|f\|_{2, M(2)}^{2}=\int_{M(2)}|f(u)|^{2} d u=\int_{0}^{\infty} \sum_{j, k=-\infty}^{\infty}\left|T_{f}(R ; j, k)\right|^{2} R d R .
$$

Given any countably infinite matrix-valued function on the positive reals, $M(R)$, we define the left multiplier operator induced by $M(R)$ to be the operator which maps $f \in L^{p}(M(2)) \cap L^{2}(M(2))$ to $M f$ where

$$
M f\left(r e^{i \phi}, \psi\right)=\frac{1}{2 \pi} \int_{0}^{\infty} \operatorname{tr}\left[M(R) T_{f}(R) J(\phi, R r, \psi)\right] R d R .
$$

Such operators commute with left multiplication by elements of $M(2)$. The following characterization of multiplier operators which are bounded on $L^{2}(M(2))$ is a consequence of the Plancherel formula for $M(2)$ : A multiplier, $M(R)$, defines a bounded multiplier operator on $L^{2}(M(2))$ if and only if the operator norm of $M(R)$ on $l^{2}(C)$ is essentially bounded on $(0, \infty)$.

We shall use the following notation to simplify the study of multiplier operators on $M(2)$. Let $D^{0} M(R, j, k)$ denote $M(R, j, k)$; let $D^{1} M(R, j, k)$ denote either $(d / d R) M(R, j, k)$ or $M(R, j+1, k+1)-M(R, j, k)$ where the difference is replaced by 0 if $k=-1,0$; let $D^{2} M(R, j, k)$ denote either $\left(d^{2} / d R^{2}+R^{-1} d / d R-((j-k) / R)^{2}\right) M(R, j, k)$ or $M(R, j+1, k+1)-$ $2 M(R, j, k)+M(R, j-1, k-1)$ where the difference is replaced by 0 if $k=-1,0,1$.

A group which is closely related to $M(2)$ is the locally compact abelian group, the direct product of the plane with the circle, denoted by $R^{2} \times T$. The dual group to $R^{2} \times T$ is the direct product of the plane with the integers, $R^{2} \times Z$. The Fourier transform of a function, $f$, defined on $R^{2} \times T$ is

$$
T_{f}(x, j)=\int_{-\pi}^{\pi} f(\hat{x}, \theta) e^{-i j \theta} d \theta
$$

where $x \in R^{2}$ and $j \in Z$. Multiplier operators on $L^{2}\left(R^{2} \times T\right) \cap$ $L^{p}\left(R^{2} \times T\right)$ are induced by functions $M(x, j)$ on $R^{2} \times Z$ by setting

$$
M f(z, \phi)=\sum_{j=-\infty}^{\infty} \int_{R^{2}} M(x, j) T_{f}(x, j) e^{i x \cdot z} e^{i j \phi} d x .
$$

As in the case of $M(2)$, the following notation will simplify the study of multiplier operators on $R^{2} \times T$. Let $D^{0} M\left(R e^{i \theta}, k\right)$ denote $M\left(R e^{i \theta}, k\right)$; let $D^{1} M\left(R e^{i \theta}, k\right)$ denote either

$$
(\partial / \partial R) M\left(R e^{i \theta}, k\right)
$$




$$
M\left(R e^{i \theta}, k+1\right)-M\left(R e^{i \theta}, k\right)
$$

where the difference is replaced by 0 if $k=-1,0$; let $D^{2} M\left(R e^{i \theta}, k\right)$ denote either

$$
\left(\partial^{2} / \partial R^{2}+R^{-1} \partial / \partial R+R^{-2} \partial^{2} / \partial \theta^{2}\right) M\left(R e^{i \theta}, k\right)
$$

or

$$
M\left(R e^{i \theta}, k+1\right)-2 M\left(R e^{i \theta}, k\right)+M\left(R e^{i \theta}, k-1\right)
$$

where the difference is replaced by 0 if $k=-1,0,1$.

2. Hörmander multiplier theorems on $M(2)$ and $R^{2} \times T$. We are now in a position to announce theorems on $M(2)$ and $R^{2} \times T$ which are analogous to the classical Hörmander multiplier theorem on $R^{n}$. The classical Hörmander theorem states: Let $\alpha$ be a multi-index $\alpha=\left(\alpha_{1}, \ldots, \alpha_{n}\right),|\alpha|=\sum_{i=1}^{n} \alpha_{i}$, and let $D^{\alpha}=\partial^{|\alpha|} / \partial x_{\alpha_{1}} \cdots \partial x_{\alpha_{n}}$. Suppose $m \in L^{\infty}\left(R^{n}\right)$ satisfies $\int_{A(s)}\left|D^{\alpha} m(x)\right|^{2} d x$ $\leqslant C s^{n-2|\alpha|}$ for $0<s<\infty$ and $|\alpha| \leqslant$ the smallest integer greater than $n / 2$, where $A(s)=\{x: s \leqslant|x| \leqslant 2 s\}$ and $C$ is a constant. Then the operator mapping $f$ to $(m \hat{f})^{\sim}$ is bounded on $L^{p}\left(R^{n}\right), 1<p<\infty$ (cf. [3]). The corresponding theorem on $M(2)$ is

(1) THEOREM. Let $M$ be the bounded left multiplier operator on $L^{2}(M(2))$ induced by the matrix-valued function $M(R)$. Let $M(R, j, k)$ denote the $j, k$ entry of the matrix $M(R)$. If $M(R, j, k)$ satisfies:

(i) $\sum_{j=-\infty}^{\infty}|M(R, j, k)|^{2} \leqslant C$ when $k=-1,0,1$ with $C$ a constant independent of $R$, and

$$
\int_{\substack{R \in(0, \infty) \\ \text { s.t.s }<R+|k|<2 s}} \sum_{\substack{k=-\infty \\ j=-\infty}}^{\infty} \sum_{j=-\infty}^{\infty}\left|D^{\alpha} M(R, j, k)\right|^{2} R d R \leqslant C s^{\lambda-2 \alpha}
$$

for $\alpha=0,1,2$ and

$$
\lambda= \begin{cases}2 & \text { if } 0<s \leqslant \frac{1}{2}, \\ 3 \quad \text { if } s>\frac{1}{2}, & 0<s<\infty,\end{cases}
$$

then $M$ is bounded on $L^{p}(M(2)), 1<p \leqslant 2$, and is of weak-type 1:1.

This theorem will be proved in $\S 3$.

The extension of this result to $L^{p}(M(2))$ for $2 \leqslant p<\infty$ follows easily from the facts that: the boundedness of $M$ on $L^{p}(M(2))$ for $2 \leqslant p<\infty$ is equivalent to the boundedness of the adjoint of $M$, denoted $M^{*}$, on $L^{q}(M(2))$ for $1<q \leqslant 2$, and $M^{*}$ is the multiplier operator induced by the transpose of $M(R)$.

It is interesting to note that Theorem (1) corresponds formally to the classical Hörmander theorem for $R^{2}$ when $0<s \leqslant \frac{1}{2}$ and to the classical Hörmander theorem for $R^{3}$ when $s>\frac{1}{2}$. This phenomenon will be discussed in the proof of Theorem (1).

The analogue of the Hörmander theorem on $R^{2} \times T$ is 
(2) THEOREM. Let $M$ be the bounded multiplier operator on $L^{2}\left(R^{2} \times T\right)$ induced by the function $M\left(R e^{i \theta}, k\right)$. If $M\left(R e^{i \theta}, k\right)$ satisfies:

(i) $\int_{-\pi}^{\pi}\left|M\left(R e^{i \theta}, k\right)\right|^{2} d \theta \leqslant C$ when $k=-1,0,1$ with $C$ a constant independent of $R$, and

$$
\int_{\substack{R \in(0, \infty) \\ \text { s.t. } s<R+|k|<2 s}} \sum_{\substack{k=-\infty \\ \infty}}^{\infty} \int_{-\pi}^{\pi}\left|D^{\alpha} M\left(R e^{i \theta}, k\right)\right|^{2} d \theta R d R<C s^{\lambda-2 \alpha}
$$

for $\alpha=0,1,2$ and

$$
\lambda=\left\{\begin{array}{ll}
2 & \text { if } 0<s \leqslant \frac{1}{2}, \\
3 & \text { if } s>\frac{1}{2},
\end{array} \quad 0<s<\infty,\right.
$$

then $M$ is bounded on $L^{p}\left(R^{2} \times T\right), 1<p<\infty$, and is of weak-type 1:1.

In the remainder of this section, we shall study the correspondences between Theorems (1) and (2). If $M(R, j, k)$ defines a multiplier operator which satisfies the hypotheses of Theorem (1), call $M(R, j, k)$ a Hörmander multiplier for $M(2)$. Define Hörmander multipliers for $R^{2} \times T$ analogously.

We say that the multipliers $M(R, j, k)$ on $M(2)$ and $M\left(R e^{i \theta}, k\right)$, $\tilde{M}\left(R e^{i \theta}, k\right)$ on $R^{2} \times T$ are related if

$$
\begin{aligned}
& M(R, k, j)=\frac{1}{2 \pi} \int_{-\pi}^{\pi} M\left(R e^{i \theta}, k\right) e^{-i(k-j) \theta} d \theta, \\
& M(R, j, k)=\frac{1}{2 \pi} \int_{-\pi}^{\pi} \tilde{M}\left(R e^{i \theta}, k\right) e^{-i(k-j) \theta} d \theta .
\end{aligned}
$$

(3) Corollary. (i) Suppose the multiplier operator $M$ defined by $M(R, j, k)$ is bounded on $L^{2}(M(2))$. If the related multipliers, $M\left(R e^{i \theta}, k\right)$ and $\tilde{M}\left(R e^{i \theta}, k\right)$, are Hörmander multipliers on $R^{2} \times T$, then $M$ is bounded on $L^{p}(M(2))$, $1<p<\infty$, and is of weak-type $1: 1$.

(ii) Suppose the multiplier operator $M$ defined by $M\left(R e^{i \theta}, k\right)$ is bounded on $L^{2}\left(R^{2} \times T\right)$. If the related multiplier $M(R, j, k)$ is a Hörmander multiplier on $M(2)$, then $M$ is bounded on $L^{p}\left(R^{2} \times T\right), 1<p<\infty$, and is of weak-type $1: 1$.

The Corollary is easily proved. (i) is established by noting that the hypotheses of Theorem (2) combined with condition (a) and the Plancherel formula for $T$ imply the hypotheses of Theorem (1) for the operator induced by the transpose of $M(R)$. Similarly, the hypotheses of Theorem (2) and condition (b) imply the hypotheses of Theorem (1) for the operator induced by $M(R)$. (ii) is proved in the same manner.

3. The proof of Theorem (1). We begin the proof by applying the theory of spaces of homogeneous type [2, Chapter III]. It is easy to verify that the function

$$
n((x, \phi))= \begin{cases}{[\alpha((x, \phi))]^{3 / 2}} & \text { if } \alpha((x, \phi)) \leqslant 1 \\ \alpha((x, \phi)) & \text { otherwise }\end{cases}
$$


where $\alpha((x, \phi))=|x|^{2}+4 \sin ^{2}(\phi / 2)$, defines a pseudo-distance, $d$, in the sense of Coifman-Weiss [2, p. 66] by setting $d(u, v)=n\left(u^{-1} v\right)$. Using this function, together with Haar measure on $M(2),(2 \pi)^{-1} d x d \phi$, one can check that $M(2)$ is a space of homogeneous type. The essential step is to note that the sets $\{(x$, $\left.\phi) \in M(2):|x|^{2}+4 \sin ^{2}(\phi / 2)<r\right\}$ have Haar measure on the order of $r^{3}$ if $r$ is small and on the order of $r^{2}$ if $r$ is large. Thus, the sets $S_{r}=$ $\{(x, \phi) \in M(2): d((x, \phi),(0,0))<r\}$ have measure on the order of $r$. The work of Coifman, de Guzman, and Weiss [1], [2] shows that this property of the sets $S_{r}$ allows one to prove analogues of the classical Whitney and Wiener covering lemmas. These lemmas, in turn, may be used to obtain a CalderónZygmund theory of integral operators on $M(2)$. It is the condition on the measure of the sets $S_{r}$ that requires the two part definition of $n$ which results in the Hörmander theorem on $M(2)$ corresponding in part to the Hörmander theorem on $R^{3}$ and in part to the Hörmander theorem on $R^{2}$.

The next step in the proof is to combine the existence of a well-behaved approximation to the identity on $M(2)$ with the Calderón-Zygmund theory to obtain a criterion for the $L^{p}$ boundedness of operators which map $L^{2}$ to itself and commute with the group operation.

The proper approximate identity is given for $(x, \phi) \in M(2)$ and $r$ a positive real number by

$$
G((x, \phi), r)= \begin{cases}W\left(x, r^{2 / 3}\right) \tilde{w}\left(\phi, r^{2 / 3}\right), & \text { if } 0<r \leqslant 1, \\ W(x, r) \tilde{w}(\phi, r), & \text { if } r>1,\end{cases}
$$

where $W$ is the Gauss kernel for $R^{2}, W(x, r)=(4 \pi r)^{-1} \exp \left(-|x|^{2} / 4 r\right)$, and $\tilde{w}$ is the periodization of the Gauss kernel for $R$,

$$
\tilde{w}(2 \pi t, r)=2 \pi \sum_{k=-\infty}^{\infty} w(t+k, r)
$$

with

$$
w(t, r)=(4 \pi r)^{-1 / 2} e^{-t^{2} / 4 r}, \quad-\frac{1}{2}<t<\frac{1}{2} .
$$

For $u \in M(2)$, set $\Gamma_{r}(u)=G(u, r)-G(u, r / 2)$. A modification of the discussion in Coifman and Weiss [2, Chapter III, $\$ \S 2,3]$ gives the following result:

(4) Theorem. Suppose $M$ is a bounded linear operator on $L^{2}(M(2))$ which commutes with the left action of $M(2)$. Suppose that there exist constants $\varepsilon$ and $C$ such that

$$
\int_{n(u)>r}\left|\left[M\left(\Gamma_{r}\right)\right](u)\right|^{2} n(u)^{1+2 \varepsilon} d u \leqslant C r^{2 \varepsilon} .
$$

Then $M$ defines a bounded operator on $L^{2}(M(2)) \cap L^{p}(M(2)), 1<p \leqslant 2$, and is of weak-type $1: 1$.

We shall describe how properties of $n(u)$ combined with the Plancherel formula for $M(2)$ and Theorem (4) determine criteria for the $L^{p}$ boundedness of multiplier operators on $M(2)$. Choosing $\varepsilon=\frac{1}{6}$ when $r \leqslant 1$ and $\varepsilon=\frac{1}{2}$ when $r>1$, a calculation using the fact that 


$$
\left(|x|^{2}+4 \sin ^{2} \phi / 2\right)^{4 / 3}<\left(|x|^{2}+4 \sin ^{2} \phi / 2\right)^{2} \quad \text { if } n((x, \phi))>1,
$$

shows that (d) is implied by

$$
\int_{M(2)}\left|\left[M\left(\Gamma_{r}\right)\right](u)\right|^{2}|\alpha(u)|^{2} d u \leqslant C \begin{cases}r^{1 / 3} & \text { if } r \leqslant 1, \\ r & \text { if } r>1 .\end{cases}
$$

Applying the Plancherel formula for $M(2)$ to this, we see that (d) is implied by

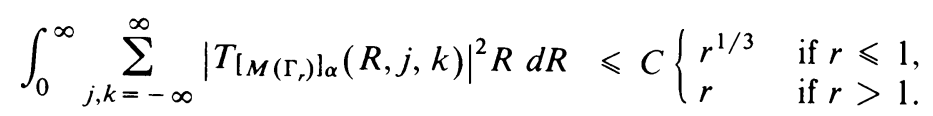

In order to study this expression, we write the Fourier transform of the product of a function $f$ on $M(2)$ with $\alpha$ in terms of the Fourier transform of $f$.

$$
\begin{aligned}
T_{f \alpha}(R, j, k)= & -\Delta^{2} T_{f}(R, j-1, k-1)-\left(\frac{\partial^{2}}{\partial R^{2}}+\frac{1}{R} \frac{\partial}{\partial R}\right) T_{f}(R, j, k) \\
& +\left(\frac{k-j}{R}\right)^{2} T_{f}(R, j, k)
\end{aligned}
$$

where $\Delta^{2}$ is the second difference operator:

$$
\begin{aligned}
\Delta^{2} T_{f}(R, j-1, k-1)= & T_{f}(R, j+1, k+1) \\
& -2 T_{f}(R, j, k)+T_{f}(R, j-1, k-1) .
\end{aligned}
$$

Another calculation shows that

$$
T_{\Gamma_{r}}(R, j, k)= \begin{cases}\exp \left(-r^{2 / 3}\left(R^{2}+k^{2}\right)\right)-\exp \left(-\left(\frac{r}{2}\right)^{2 / 3}\left(R^{2}+k^{2}\right)\right) \\ \operatorname{lif} k=j \text { and } r \leqslant 1, \\ \exp \left(-r\left(R^{2}+k^{2}\right)\right)-\exp \left(-\frac{r}{2}\left(R^{2}+k^{2}\right)\right) \\ \text { if } k=j \text { and } r>1,\end{cases}
$$

Thus the matrix $T_{\Gamma_{r}}(R)$ is diagonal.

Assume the hypotheses of Theorem (1). We will have proved the theorem provided we show (e) holds. In order to do this, recall Leibnitz's rule for second differences: if

$$
\Delta^{1}(A(j, k))=A(j+1, k+1)-A(j, k)
$$

and

$$
\Delta^{2}(A(j, k))=A(j+2, k+2)-2 A(j+1, k+1)+A(j, k),
$$

then

$$
\begin{aligned}
& \Delta^{2}[A(j-1, k-1) B(j-1, k-1)] \\
&= {\left[\Delta^{2} A(j-1, k-1)\right] B(j, k)+\Delta A(j-1, k-1) \Delta B(j-1, k-1) } \\
&+\Delta A(j, k) \Delta B(j, k)+A(j, k)\left[\Delta^{2} B(j-1, k-1)\right] .
\end{aligned}
$$


Applying the above work, as well as the familiar Leibnitz's rule, to (e) gives

$$
\begin{aligned}
& \int_{0}^{\infty} \sum_{j, k=-\infty}^{\infty} \mid-\left[\left(\frac{\partial^{2}}{\partial R^{2}}+\frac{1}{R} \frac{\partial}{\partial R}\right) M(R, j, k)\right] T_{\Gamma_{r}}(R, k, k) \\
& -2 \frac{\partial}{\partial R} M(R, j, k) \frac{\partial}{\partial R} T_{\Gamma_{r}}(R, k, k) \\
& -M(R, j, k)\left(\frac{\partial}{\partial R^{2}}+\frac{1}{R} \frac{\partial}{\partial R}\right) T_{\Gamma_{r}}(R, k, k) \\
& +\left(\frac{k-j}{R}\right)^{2} M(R, j, k) T_{\Gamma_{r}}(R, k, k) \\
& -\left[\Delta^{2} M(R, j-1, k-1)\right] T_{\Gamma_{r}}(R, k, k) \\
& \text { - } \Delta M(R, j-1, k-1) \Delta T_{\Gamma_{r}}(R, k-1, k-1) \\
& \text { - } \Delta M(R, j, k) \Delta T_{\Gamma_{r}}(R, k, k) \\
& -\left.M(R, j, k) \Delta^{2} T_{\Gamma_{r}}(R, k-1, k-1)\right|^{2} R d R \\
& \leqslant \begin{cases}C r^{1 / 3} & \text { if } r \leqslant 1 \\
C r & \text { if } r>1\end{cases}
\end{aligned}
$$

The remainder of the proof consists of showing that the conditions in the hypotheses of Theorem (1) correspond to the terms on the left-hand side of (f) being dominated by $C r^{1 / 3}$ if $r \leqslant 1$, and $C r$ if $r>1$.

As an example of the method of establishing the required inequalities, consider

$$
I=\int_{0}^{\infty} \sum_{j, k=-\infty}^{\infty}\left|D^{2} M(R, j, k)\right|^{2}\left|T_{\Gamma_{r}}(R, k, k)\right|^{2} R d R .
$$

Suppose $0<r \leqslant 1$. Let $q$ be an integer $\geqslant 1$ such that $2^{-q+1} r^{-1 / 2} \leqslant 1$ $<2^{-q+2} r^{-1 / 2}$. Define $A(s)=\{(R, k): 0<R<\infty, k$ is an integer, $s \leqslant R+|k|<2 s\}$. Then we may decompose $I$ as

$$
\begin{aligned}
I= & \left.\sum_{n=q}^{\infty} \int_{\substack{R \in(0, \infty) \\
\text { s.t. }(R, k) \in A}} \sum_{\substack{k=-\infty \\
\left(2^{-n_{r}-1 / 2}\right)}}^{\infty}+\sum_{n=-q+1}^{\infty} \int_{\substack{R \in(0, \infty) \\
\text { s.t. }(R, k) \in A}} \sum_{\substack{k=-\infty \\
\left(2^{n_{r}-1 / 2}\right)}}^{\infty}\right) \\
& \cdot \sum_{j=\infty}^{\infty}\left|D^{2} M(R, j, k)\right|^{2}\left|T_{\Gamma_{r}}(R, k, k)\right|^{2} R d R=I_{1}+I_{2} .
\end{aligned}
$$


Note that if $n \geqslant q$, then $(R, k) \in A\left(2^{-n} r^{-1 / 2}\right)$ implies $k=0$. A computation shows that $\left|T_{\Gamma_{r}}(R, k, k)\right| \leqslant C r^{2 / 3}\left(R^{2}+k^{2}\right)$ if $r<1$. This, combined with the hypothesis of Theorem (1) for $\alpha=2, \lambda=2$, implies that

$$
\int_{2^{-n_{r}-1 / 2}}^{2^{-n+1} r^{-1 / 2}} \sum_{j=-\infty}^{\infty}\left|D^{2} M(R, j, 0)\right|^{2}\left|T_{\Gamma_{r}}(R, 0,0)\right|^{2} R d R \leqslant C 2^{-2 n+4} r^{1 / 3},
$$

and hence that

$$
I_{1} \leqslant \sum_{n=q}^{\infty} C 2^{-2 n+4} r^{1 / 3} \leqslant C r^{1 / 3} .
$$

Similarly, if $n \geqslant-q+1$ (i.e. $1 / 2<2^{n} r^{-1 / 2}$ ), the above estimate of $T_{\Gamma,}(R, k, k)$ combined with the hypothesis of Theorem (1) for $\alpha=2, \lambda=3$ implies that

$$
\int_{\substack{R \in(0, \infty) \\ \text { s.t. }(R, k) \in A\left(2^{n} r^{-1 / 2}\right)}} \sum_{\substack{k=-\infty \\ k=-\infty}}^{\infty}\left|D^{2} M(R, j, k)\right|^{2}\left|T_{\Gamma_{r}}(R, k, k)\right|^{2} R d R \leqslant C 2^{3 n+2} r^{1 / 3} .
$$

The quantity on the left side of this inequality is also dominated by $C 2^{-n} r^{1 / 2}$, as can be checked by noting that $\left|T_{\Gamma_{r}}(R, k, k)\right| \leqslant C$ and using the hypothesis of Theorem (1) for $\alpha=2, \lambda=3$. Combining the last two estimates gives

$$
I_{2} \leqslant C\left(\sum_{n=-q+1}^{0} 2^{3 n+2} r^{1 / 3}+\sum_{n=0}^{\infty} 2^{-n} r^{1 / 2}\right) \leqslant C r^{1 / 3} .
$$

Thus, we have shown that $I=I_{1}+I_{2} \leqslant C r^{1 / 3}$ if $r \leqslant 1$. The case when $r>1$ is proved in the same manner.

Similar arguments using the following estimates prove the required inequalities for the other terms on the left side of ( $f$ ).

$$
\begin{gathered}
\left|\frac{d}{d R} T_{\Gamma_{r}}(R, k, k)\right| \leqslant C\left\{\begin{array}{l}
r^{\delta} R, \\
R /\left(R^{2}+k^{2}\right),
\end{array}\right. \\
\left|\left(\frac{d^{2}}{d R^{2}}+\frac{1}{R} \frac{d}{d R}\right) T_{\Gamma_{r}}(R, k, k)\right| \leqslant C\left\{\begin{array}{l}
r^{\delta}, \\
1 /\left(R^{2}+k^{2}\right),
\end{array}\right. \\
\left|\Delta T_{\Gamma_{r}}(R, k, k)\right| \leqslant C \begin{cases}r^{\delta}|k| & \text { if } k \neq 0, \\
|k| /\left(R^{2}+k^{2}\right) & \text { if } k \neq-1,0, \\
r^{\delta} & \text { if } k=0,\end{cases} \\
\left|\Delta^{2} T_{\Gamma_{r}}(R, k, k)\right| \leqslant C \begin{cases}r^{\delta}, \\
1 /\left(R^{2}+k^{2}\right) & \text { if } k \neq-1,0,1, \\
r^{2 / 3} \exp \left(-r^{2 / 3} R^{2}\right) & \text { if } k \neq-1,0,1 \text { and } r \leqslant 1 .\end{cases}
\end{gathered}
$$

In the above inequalities, $\delta=\frac{2}{3}$ if $r \leqslant 1$ and $\delta=1$ if $r>1$.

Theorem (2) may be proved by the same arguments used to prove Theorem (1); details will be omitted. 
4. Applications. The Laplace operator, $\nabla^{2}$, on $M(2)$ is given by

$$
\nabla^{2} f\left(r e^{i \phi}, \psi\right)=\left(\frac{\partial^{2}}{\partial r^{2}}+\frac{1}{r} \frac{\partial}{\partial r}+\frac{1}{r^{2}} \frac{\partial^{2}}{\partial \phi^{2}}+\frac{\partial^{2}}{\partial \psi^{2}}\right) f\left(r e^{i \phi}, \psi\right) \text {. }
$$

A computation shows that the multiplier

$$
M(R, j, k)= \begin{cases}R^{2}+j^{2} & \text { if } j=k \\ 0 & \text { if } j \neq k\end{cases}
$$

corresponds to the differential operator $-\nabla^{2}$. We are led to define the fractional Laplace operator on $M(2)$ to be the multiplier operator defined by

$$
M(R, j, k)= \begin{cases}\left(R^{2}+j^{2}\right)^{i \lambda} & \text { if } j=k \\ 0 & \text { if } j \neq k\end{cases}
$$

for real $\lambda$. This multiplier satisfies the hypotheses of Theorem (1) and since it is diagonal, the induced multiplier operator is selfadjoint, so that we may conclude that the fractional Laplace operator on $L^{p}(M(2))$ is bounded for $1<p<\infty$ and is of weak-type $1: 1$.

Next, we illustrate how the preceding work can be used to transfer basic results on three-dimensional Euclidean space to $M(2)$. Consider the Riesz kernels

$$
r_{j}\left(x_{1}, x_{2}, x_{3}\right)=\pi^{-2} x_{j}\left(x_{1}^{2}+x_{2}^{2}+x_{3}^{2}\right)^{-2}, \quad j=1,2,3 .
$$

Set $r\left(x_{1}, x_{2}, x_{3}\right)=r_{1}\left(x_{1}, x_{2}, x_{3}\right)+i r_{2}\left(x_{1}, x_{2}, x_{3}\right)$. Let $\rho$ be the function on $R^{2} \times T$ induced from $r$ by periodizing with respect to $x_{3}: \rho\left(x_{1}, x_{2}, \psi\right)$ $=\sum_{k=-\infty}^{\infty} r\left(x_{1}, x_{2}, \psi+2 \pi k\right)$, i.e.

$$
\rho\left(R e^{i \theta}, \psi\right)=\pi^{-2} \sum_{k=-\infty}^{\infty} R e^{i \theta}\left(R^{2}+(\psi+2 \pi k)^{2}\right)^{-2} .
$$

The multiplier on $R^{2} \times T$ defined by $\rho$ is

$$
T_{\rho}\left(R e^{i \theta}, j\right)=\pi^{-2} R e^{i \theta}\left(R^{2}+j^{2}\right)^{-1 / 2}=M\left(R e^{i \theta}, j\right) .
$$

This multiplier is a Hörmander multiplier on $R^{2} \times T$. Applying Corollary (3) to this multiplier shows that the matrix $M(R)$ with $j, k$ entry

$$
\begin{aligned}
M(R, j, k) & =(2 \pi)^{-1} \int_{-\pi}^{\pi} M\left(R e^{i \theta}, k\right) e^{-i(k-j) \theta} d \theta \\
& = \begin{cases}\pi^{-2} R\left(R^{2}+k^{2}\right)^{-1 / 2} & \text { if } k=j-1, \\
0 & \text { otherwise }\end{cases}
\end{aligned}
$$

is a Hörmander multiplier on $M(2)$. Clearly the transpose of $M(R)$ is also a Hörmander multiplier on $M(2)$. Thus $M(R)$ defines a bounded multiplier operator on $L^{p}(M(2)), 1<p<\infty$, which is of weak-type $1: 1$.

Another method of obtaining this result is to note that the multiplier operator induced by $M(R, j, k)$ on $M(2)$ is defined by the $M(2)$ convolution operator with kernel $\rho\left(R e^{i \theta}, \psi\right)$. Further, it is not hard to check that if $k\left(R e^{i \theta}, \psi\right)$ is a set function on $R^{2} \times T$ with the property that 
$e^{i \xi} k\left(R e^{i \theta}, \psi\right)=k\left(R e^{i(\theta+\xi)}, \psi\right)$, then $k$ defines a bounded left convolution operator on $L^{p}(M(2))$ if and only if $k$ defines a bounded convolution operator on $L^{p}\left(R^{2} \times T\right), 1<p<\infty$. The $L^{p}$ boundedness of the multiplier operator on $M(2)$ then follows from the Hörmander theorem on $R^{2} \times T$ and these observations.

\section{REFERENCES}

1. R. R. Coifman and M. de Guzman, Singular integrals and multipliers on homogeneous spaces, Rev. Un. Mat. Argentina 25 (1970/71), 137-143. MR 47 \#180.

2. R. Coifman and G. Weiss, Analyse harmonique non-commutative sur certains espaces homogènes étude de certaines intégrales singulières, Lecture Notes in Math., vol. 242, SpringerVerlag, Berlin and New York, 1971.

3. L. Hörmander, Estimates for translation invariant operators in $L^{p}$ spaces, Acta Math. 104 (1960), 93-140. MR 22 \# 12389.

4. R. Rubin, Harmonic analysis on the group of rigid motions of the euclidean plane, Studia Math. (to appear).

Department of Mathematical Sciencis, Florida International University, Miami, FLORIDA 33199 